\title{
Drug-mimicking nanofibrous peptide hydrogel for inhibition of inducible nitric oxide synthase
}

\author{
David G. Leach ${ }^{1}$, Jared M. Newton ${ }^{2,3}$, Marcus A. Florez, ${ }^{2,3}$,Tania L. Lopez-Silva ${ }^{1}$, Adrianna A. Jones ${ }^{1}$, \\ Simon Young ${ }^{4}$, Andrew G. Sikora*2, Jeffrey D. Hartgerink*1 \\ ${ }^{1}$ Department of Chemistry, Department of Bioengineering, Rice University, Houston, TX 77005. \\ ${ }^{2}$ Department of Otolaryngology-Head and Neck Surgery, Baylor College of Medicine, Houston, TX 77030. \\ ${ }^{3}$ Interdepartmental Program in Translational Biology and Molecular Medicine, Baylor College of Medicine, Houston, TX \\ 77030. \\ ${ }^{4}$ Department of Oral \& Maxillofacial Surgery, University of Texas Health Science Center, Houston, TX 77054.
}

\section{SUPPORTING INFORMATION}

\section{Expanded peptide synthesis and purification methods}

Peptide synthesis reagents were purchased from EMD Chemicals (Philadelphia, PA). A combination of manual synthesis and an Apex Focus XC (Aapptec) synthesizer was used to synthesize the multi-domain peptides $\mathrm{K}_{2}(\mathrm{SL})_{6} \mathrm{~K}_{2}, \mathrm{E}_{2}(\mathrm{SL})_{6} \mathrm{E}_{2}, \mathrm{R}_{2}(\mathrm{SL})_{6} \mathrm{R}_{2}$ and the precursor to the L-NIL MDP, $\mathrm{K}^{\mathrm{Mmt}}{ }_{2}(\mathrm{SL})_{6} \mathrm{~K}^{\mathrm{Mmt}}{ }_{2}$ according to a standard synthetic method previously published. ${ }^{21}$ To briefly summarize, each synthesis was performed using low loading Rink Amide MBHA resin (0.32 mmol/g). All amino acid were dissolved in 1:1 DMF:DMSO, and 20\% piperidine in 1:1 DMF:DMSO was used to for deprotection reactions. Coupling steps were performed by mixing 4 equivalents amino acid, 4 equivalents of HATU (1-[Bis(dimethylamino)methylene]-1H-1,2,3-triazolo[4,5b]pyridinium 3-oxid hexafluorophosphate), and 6 equivalents of DiEA (diisoproylethylamine), followed by shaking for 45 minutes. Upon completion of the syntheses the N-termini were acetylated by shaking the resin for 45 min with 5 equivalents of acetic anhydride mixed with 6 equivalents of DiEA in DCM, repeated once.

After acetylation and before cleavage of the peptide from the resin, the following method was used to convert $\mathrm{K}^{\mathrm{Mmt}}{ }_{2}(\mathrm{SL})_{6} \mathrm{~K}^{\mathrm{Mmt}}{ }_{2}$ on resin to the L-NIL MDP. Deprotection of the lysine side chains was achieved by removal of the Mmt protection groups with four successive 15 min washes of AcOH/TFE/DCM (1:2:7), washing until the yellow color of released trityl cation was no longer observed. After washing the deprotected resin with DCM, a reaction mixture was added containing 12 equivalents ethyl acetimidate- $\mathrm{HCl}$ and 24 equivalents DiEA in DCM, and the mixture was shaken for 3 hours. The reaction was then drained, a second reaction mixture added, and shaken again for 2 hours, followed by drainage and a third fresh reaction mixture added and shaken for 1 hour. This sequence was done to ensure maximum conversion of the lysine side chains to L-NIL functional groups.

Cleavage of peptides achieved by shaking for 3 hours with TFA and protecting scavengers, in a 1:1:1:1:18 ratio of Milli-Q water : triisopropylsilane (TIPS) : anisole : ethane dithiole (EDT) : TFA. Rotary evaporation was used to remove excess TFA, and trituration with diethyl ether yielded crude peptide that was further washed with diethyl ether, dried, and purified by dialysis against Milli-Q water for 5-7 days using 100-500 D MWCO dialysis tubing (Spectra/Por, Spectrum Laboratories Inc., Rancho Dominguez, CA). Dialyzed peptide solutions were adjusted to $\mathrm{pH}$ 7.2-7.4, after which they were sterile filtered using $0.2 \mu \mathrm{m}$ filters and lyophilized to powder for storage and use in experiments. All peptides were analyzed by Autoflex MALDI-TOF MS (Bruker Instruments, Billerica, MA) for confirmation of successful synthesis (Figure S1). 

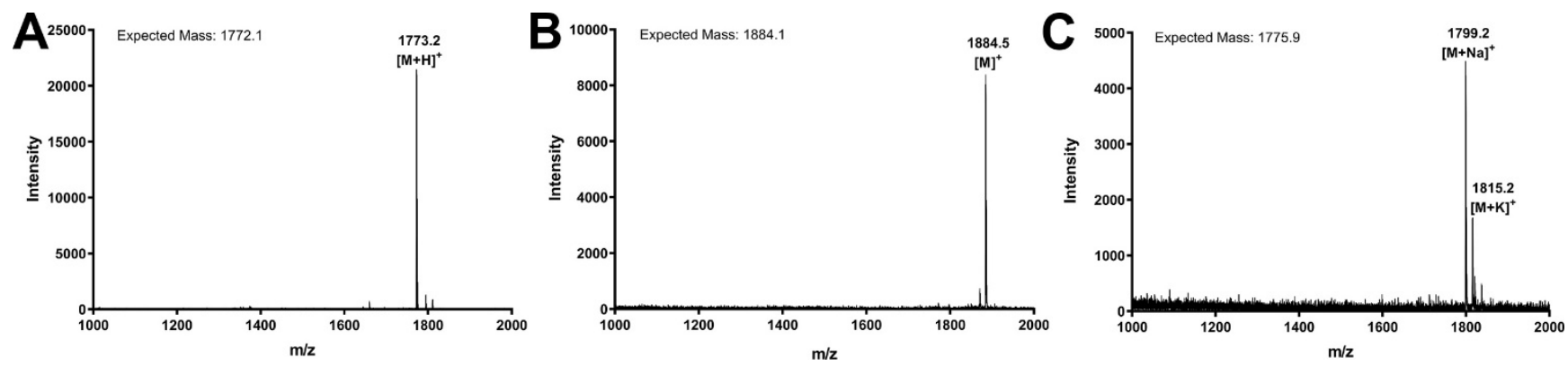

Figure S1. MALDI-TOF mass spectroscopy spectra for synthesized MDPs (A) $\mathrm{K}_{2}(\mathrm{SL})_{6} \mathrm{~K}_{2},(\mathrm{~B}) \mathrm{R}_{2}(\mathrm{SL})_{6} \mathrm{R}_{2},(\mathrm{C})$ $\mathrm{E}_{2}(\mathrm{SL})_{6} \mathrm{E}_{2}$.

Table S1. Antibodies used for immunostaining protocols.

\begin{tabular}{|c|c|c|c|c|}
\hline Primary Antibody & Dilution & Source & Catalog \# & Secondary Antibody \\
\hline $\begin{array}{l}\text { Rabbit anti- } \\
\text { Nitrotyrosine }\end{array}$ & $1: 200$ & $\begin{array}{l}\text { Millipore- } \\
\text { Sigma }\end{array}$ & $06-284$ & Anti-Rabbit AF® 568 \\
\hline $\begin{array}{l}\text { Rabbit IgG isotype } \\
\text { control }\end{array}$ & $1: 200$ & Invitrogen & 31235 & Anti-Rabbit AF® 568 \\
\hline Rabbit anti- $\alpha$ SMA & $1: 300$ & Genetex & GTX100034 & Anti-Rabbit AF® 568 \\
\hline
\end{tabular}

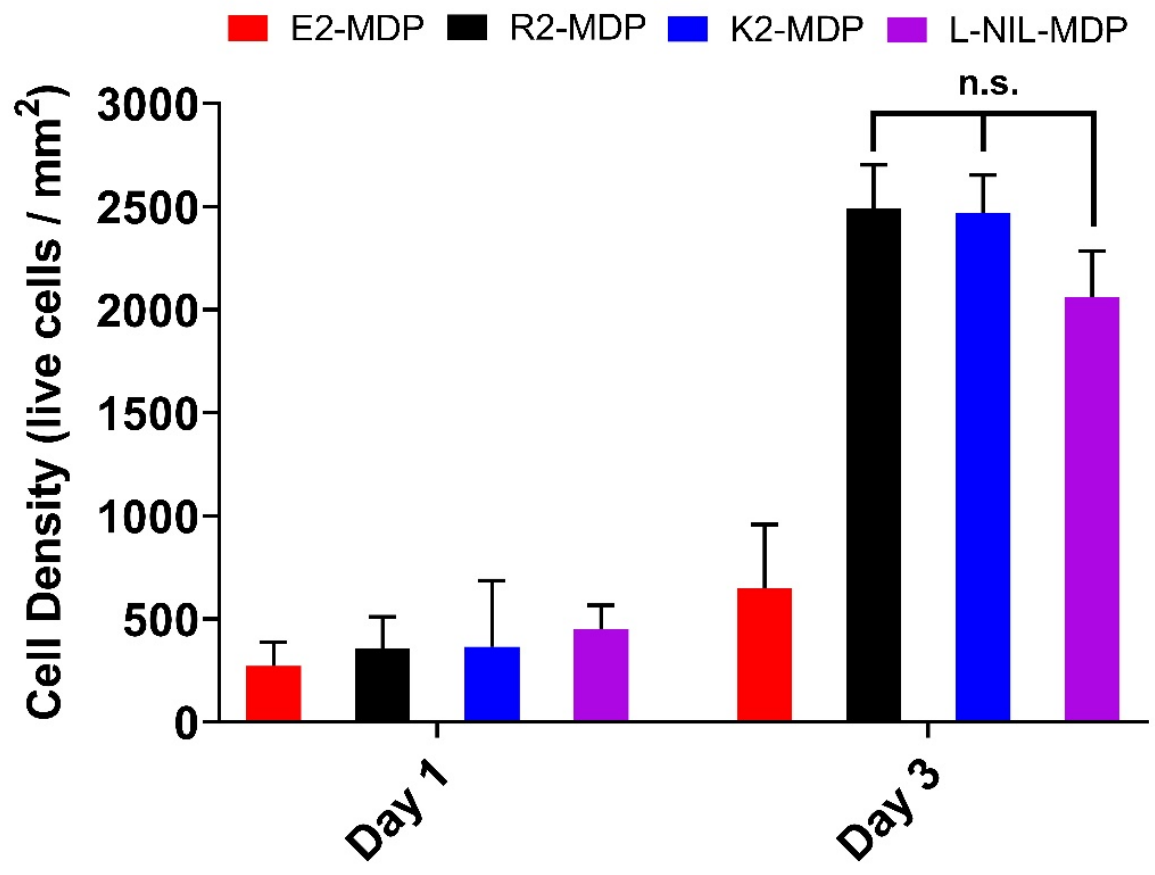

Figure S2. Quantification of live cell density cultured on MDP hydrogel surfaces in vitro to evaluate proliferation. RAW 264.7 cells were seeded on top of $70 \mu \mathrm{L}$ hydrogel pucks under $200 \mu \mathrm{L}$ of media (changed every two days) and processed under live-dead viability assays at days 1 and 3 (values are mean \pm SD with $n=3$ images). Cells are observed to not proliferate as readily on the negatively charged E2-MDP compared to the three cationic hydrogels. R2-MDP, K2-MDP, and L-NIL-MDP cell density values at day 3 show no statistically significant differences (n.s. $=\mathrm{p}>0.05$ ). 


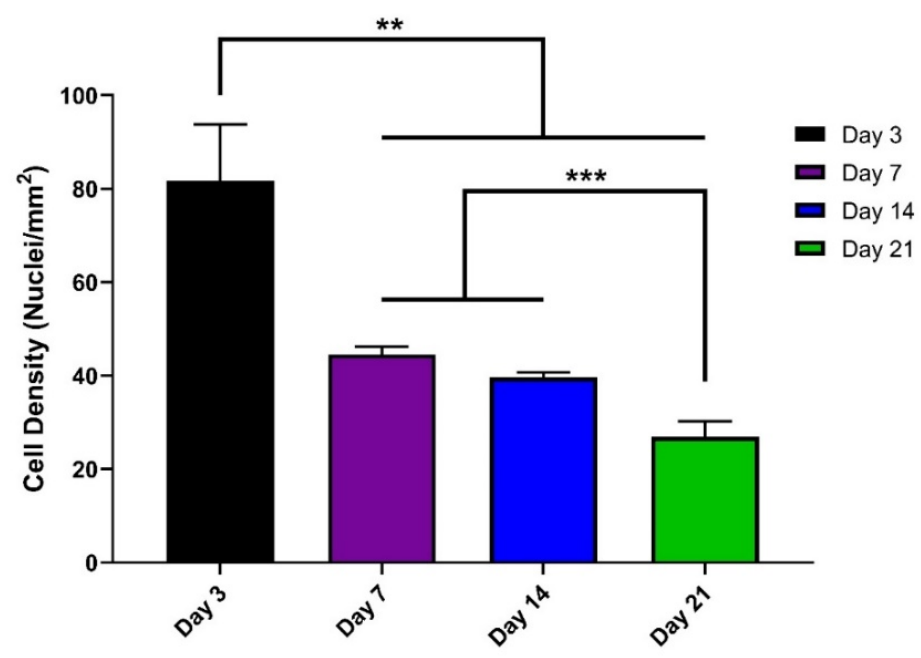

Figure S3. Quantification of cell density in vivo in subcutaneous L-NIL-MDP hydrogel implants over time, counting average number of nuclei per $\mathrm{mm}^{2}$ of tissue section. Cell density is observed to decrease from day 3 to day 21. For each data set values are mean \pm SEM with $n=3, * *=p$-value $<0.05$, and $* * *=p$-value $<0.02$.

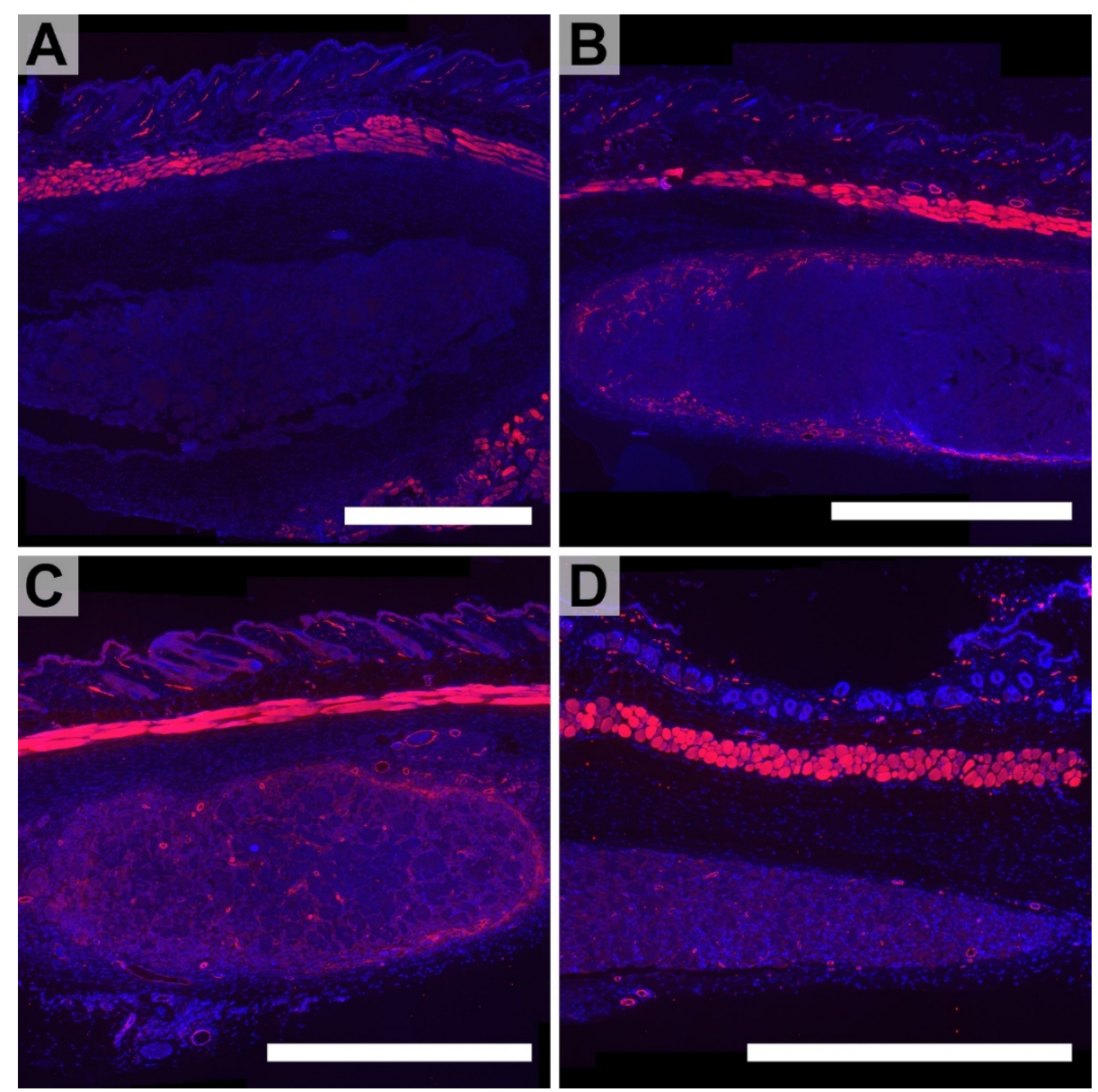

Figure S4. Blood vessel immunostaining of L-NIL-MDP hydrogel implants using anti- $\alpha$ SMA (red) and DAPI (blue) nuclei counterstain, showing some degree of small blood vessel formation in and around the implant. (A) L-NIL-MDP hydrogel 3 days post subcutaneous injection; (B) 7 days post injection; (C) 14 days post injection; (D) 21 days post injection. All scale bars $=1 \mathrm{~mm}$ (implant size in general decreases over time due to natural degradation, and thus scale is adjusted to aid visualization). 


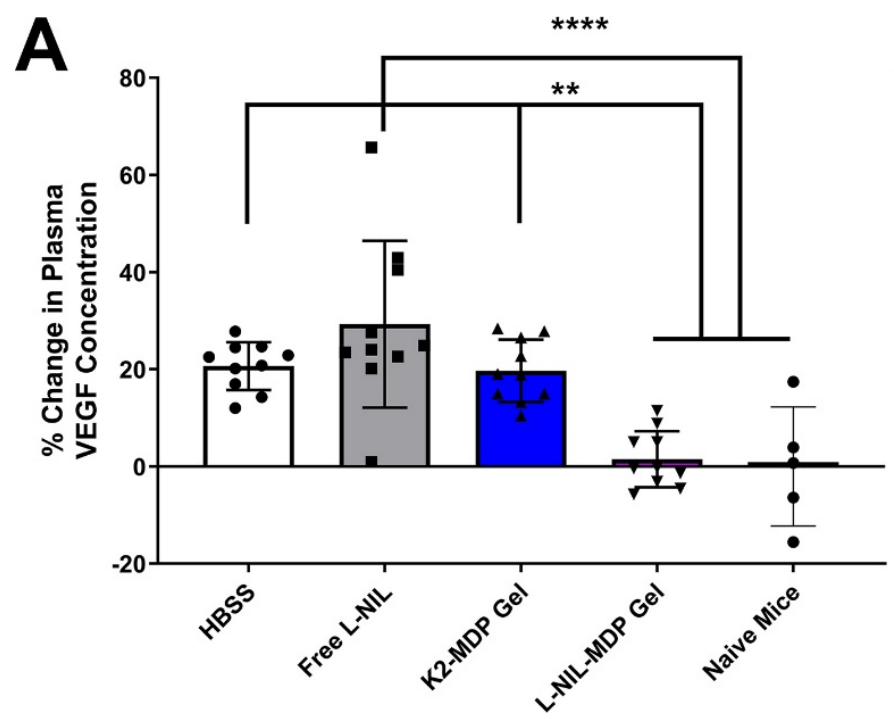

B

\begin{tabular}{|c|c|}
\hline Treatment group & $\begin{array}{c}\text { Serum VEGF level } \\
(\mathrm{pg} / \mathrm{mL})\end{array}$ \\
\hline HBSS & $142 \pm 7$ \\
Free L-NIL & $152 \pm 11$ \\
K2-MDP & $143 \pm 10$ \\
L-NIL-MDP & $112 \pm 8$ \\
Naïve & $113 \pm 17$ \\
\hline
\end{tabular}

Figure S5. Vascular endothelial growth factor (VEGF) serum levels in mice bearing B16-F0 melanoma tumors. (A) Percent change in plasma VEGF concentration from normal levels observed in naïve mice. Data shown are 5 days after $100 \mu \mathrm{L}$ intratumoral injections of either HBSS buffer control, free L-NIL drug, K2-MDP hydrogel, or L-NIL-MDP hydrogel. In each group $\mathrm{n}=10$ (except naïve mice $\mathrm{n}=5$ ), and statistical significance is noted as $* *$ $=\mathrm{p}$ value $\leq 0.004$, and $* * * *=\mathrm{p}$ value $\leq 0.0002$ (values are mean $\pm \mathrm{SD}$ ). Significant VEGF reduction was observed in sera from mice injected with the L-NIL-MDP gel compared to all other tumor-bearing groups, restoring circulating VEGF concentrations to normal levels observed in naïve healthy mice. (B) Raw VEGF serum levels in $\mathrm{pg} / \mathrm{mL}$ representative of two experiments, with values equal to mean $\pm \mathrm{SD}(\mathrm{n}=5)$.

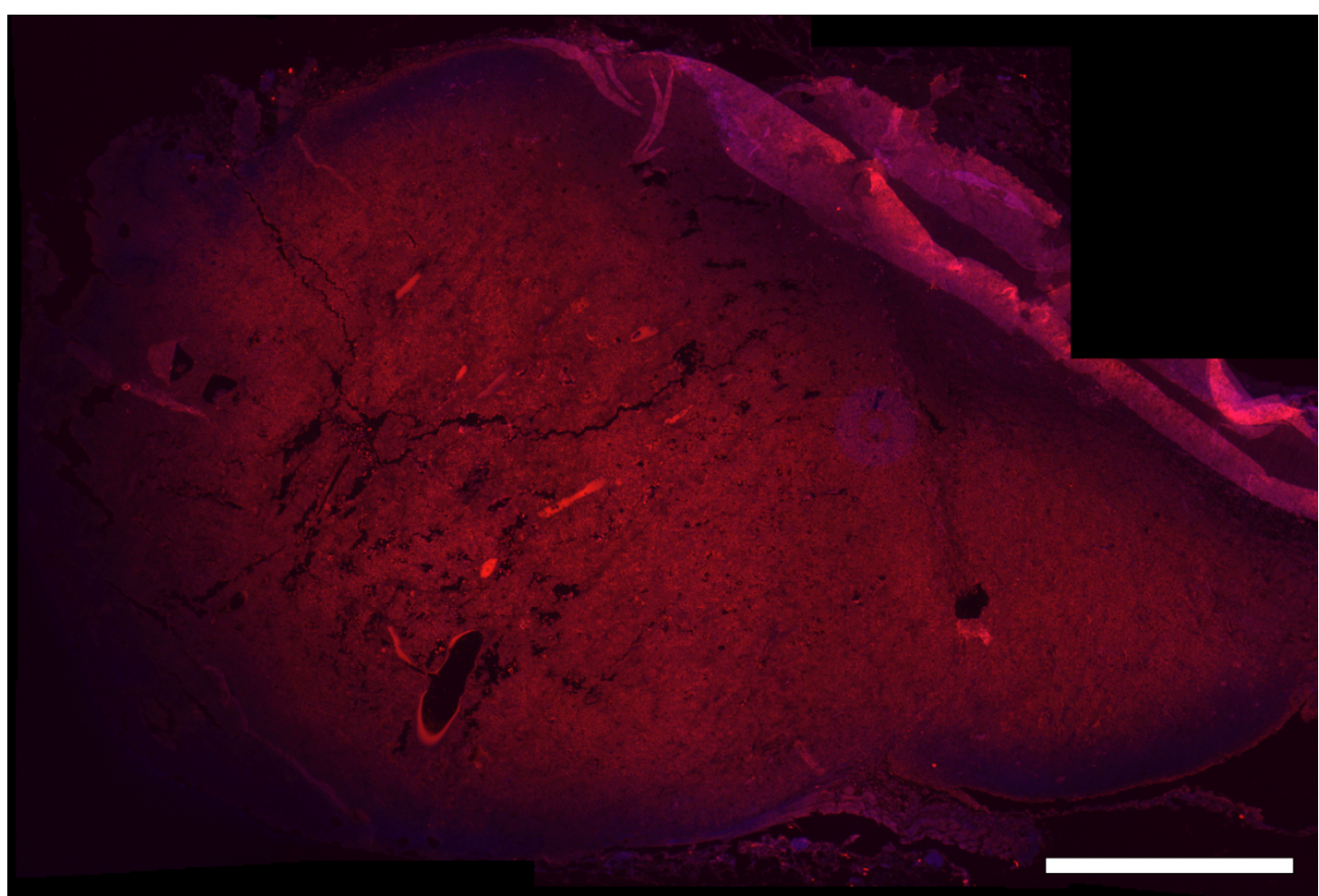

Figure S6. Anti-nitrotyrosine immunostaining of inflamed B16-F0 tumor section used as a positive control for nitrotyrosine quantification studies, using red $568 \mathrm{~nm}$ secondary antibody reactive to anti-nitrotyrosine primary and DAPI for nuclei counterstain. Scale bar is $1 \mathrm{~mm}$. 


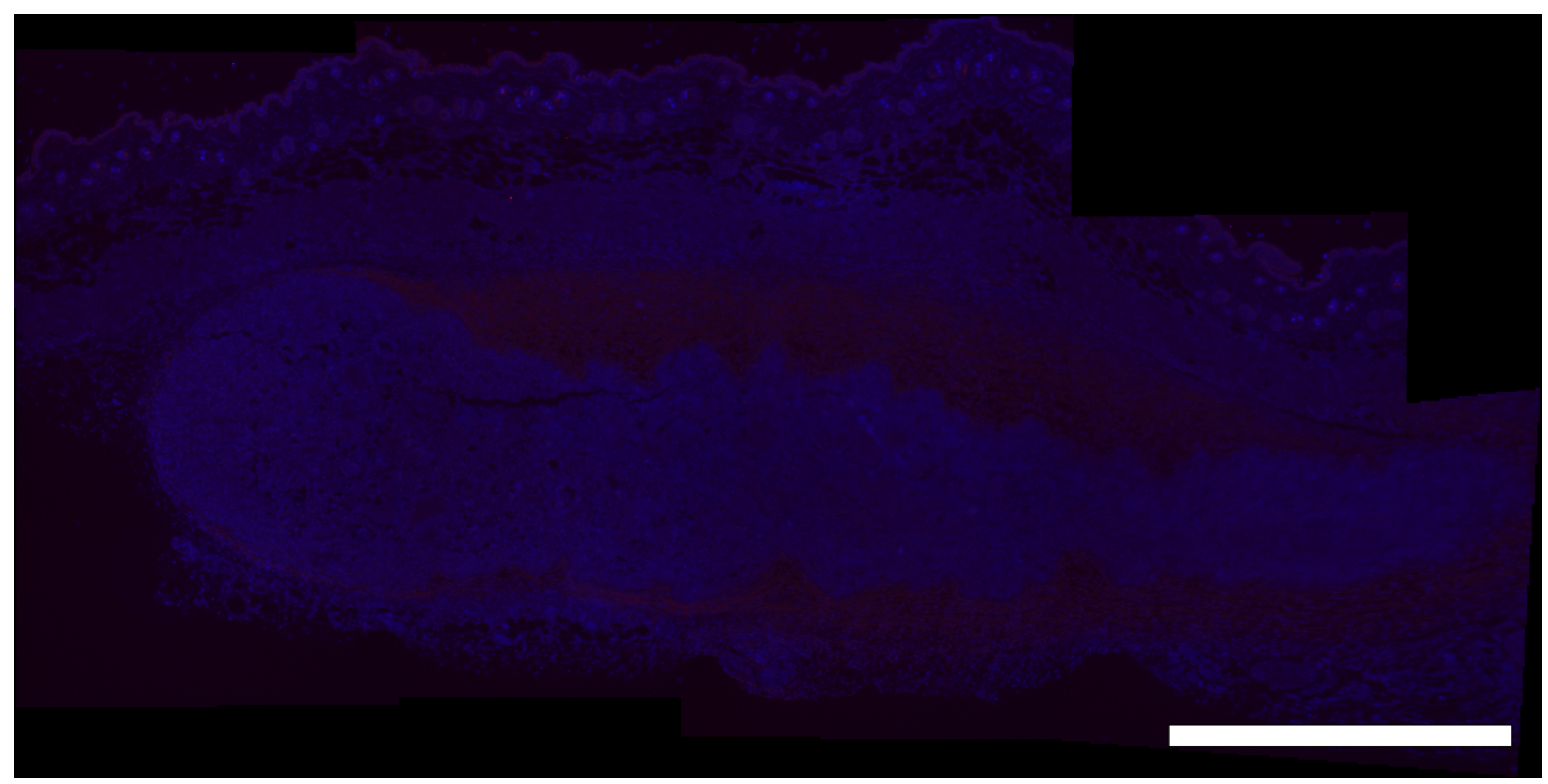

Figure S7. Day 3 L-NIL-MDP isotype control panorama for anti-nitrotyrosine immunostaining study, using rabbit IgG2a isotype control (in place of anti-nitrotyrosine primary), anti-rabbit $568 \mathrm{~nm}$ secondary, and DAPI counterstain. Scale bar is $1 \mathrm{~mm}$.

Figure S8. Day 3 K2-MDP isotype control panorama for anti-nitrotyrosine immunostaining study, using rabbit IgG2a isotype control, anti-rabbit $568 \mathrm{~nm}$ secondary, and DAPI counterstain. Scale bar is $1 \mathrm{~mm}$. 


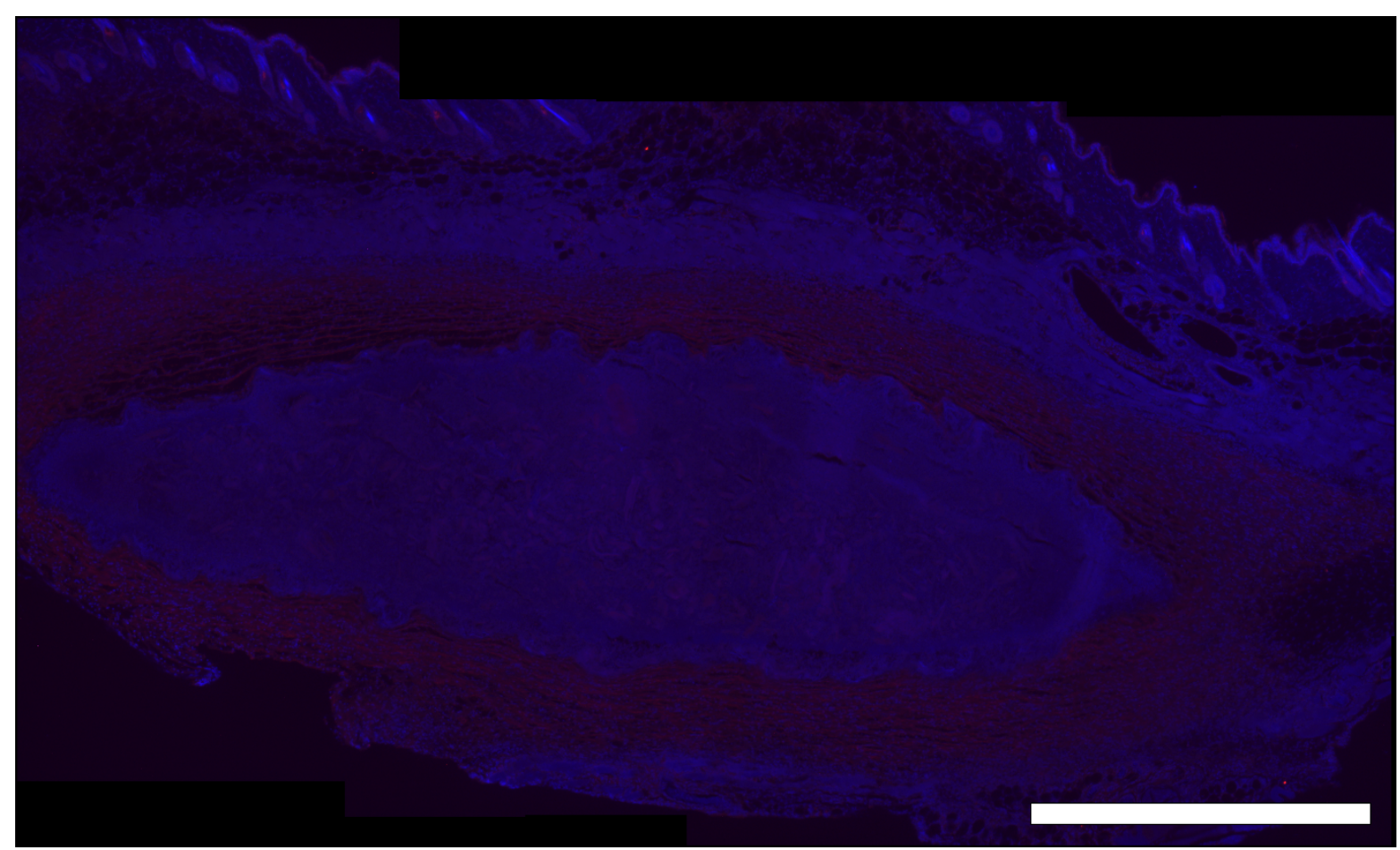

Figure S9. Day 3 R2-MDP isotype control panorama for anti-nitrotyrosine immunostaining study, using rabbit IgG2a isotype control, anti-rabbit $568 \mathrm{~nm}$ secondary, and DAPI counterstain. Scale bar is $1 \mathrm{~mm}$.

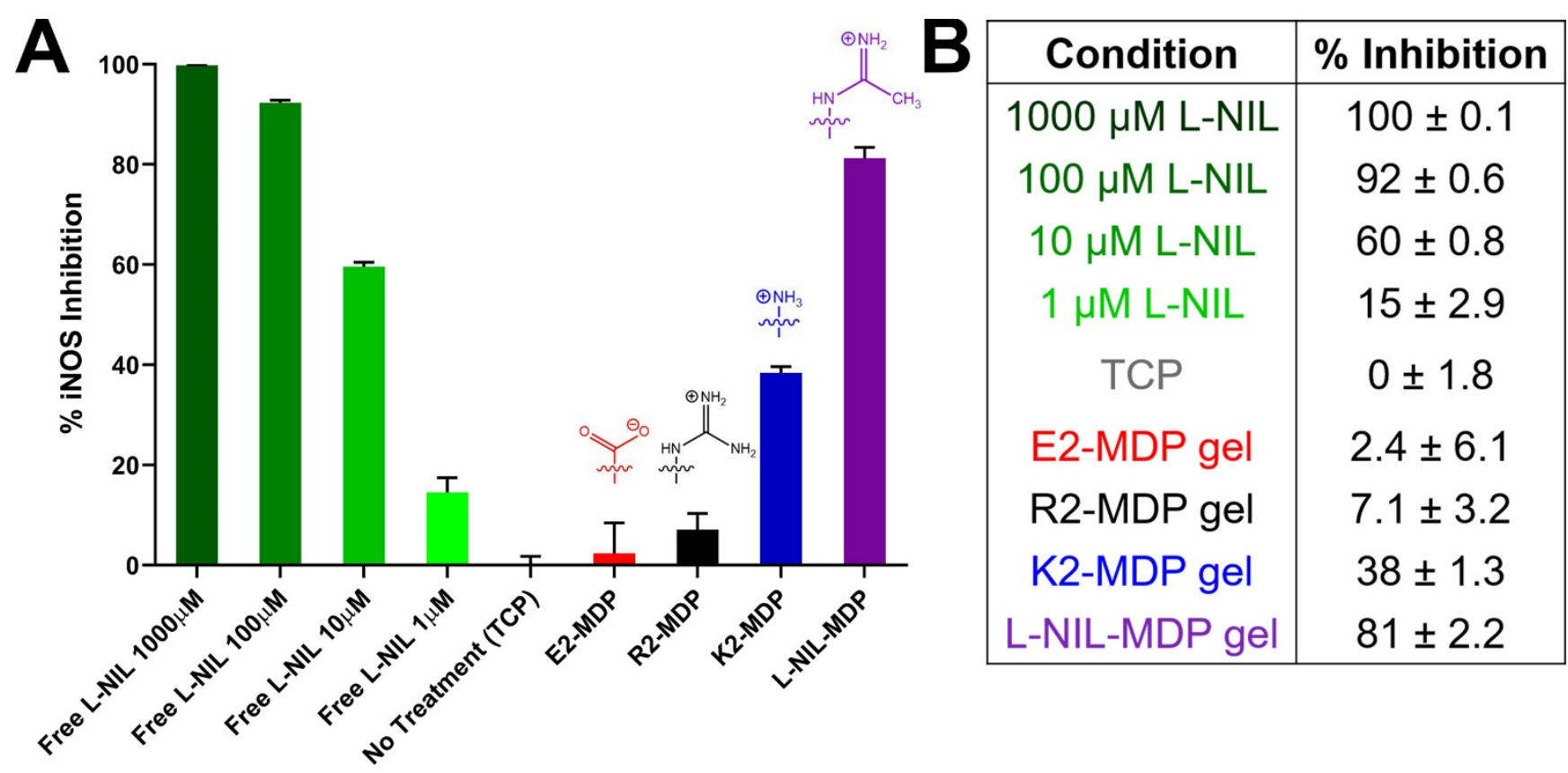

Figure S10. Raw greiss assay results used to evaluate iNOS knockdown efficacy of various materials, with data not normalized for cell viability results obtained at 24 hours. (A) Plot of \% iNOS inhibition of RAW 264.7 cells stimulated with Lipopolysaccharide (LPS) and IFN- $\gamma$ cultured in different surface conditions, showing chemical structures of key surface-exposed functional groups. (B) Quantification of relative inhibition activity; values are from two experiments and are mean \pm SD with three replicates tested per condition. 